\title{
ON THE RELIABILITY OF AN $n$-COMPONENT SYSTEM
}

\author{
Don Rawlings and Lawrence Sze
}

\begin{abstract}
$\square \quad$ Under assumptions compatible with the theory of Markov chains, we use a property of Vandermonde matrices to examine the reliability of an $n$-component system of production or service.
\end{abstract}

Keywords Markov chains; Probability theory; Stochastic processes.

\section{INTRODUCTION}

The reliability of an $n$-component system of production or service is compromised when any of its components are out of service. Viswanadham and Narahar ${ }^{[6]}$ examined the reliability of such systems in some detail. We consider a set of assumptions not pursued in Ref ${ }^{[6]}$ :

- Time, measured in discrete units, is identified with the set of positive integers. At time $t=1$, all $n$ components are in service.

- The probability of an in service (or available) component remaining in service from one time to the next is fixed and denoted by $\alpha$. For convenience, the complementary probability $1-\alpha$ is abbreviated as $\underline{\alpha}$.

- The probability of an out of service (or unavailable) component remaining out of service from one moment to the next is fixed and denoted by $\theta$. We let $\underline{\theta}=1-\theta$.

- The transition of any component between the states of being available and unavailable is independent of the other components. 
As the cases $\alpha=0=\theta$ and $\alpha=1=\theta$ are of little interest, we make the restriction $0<\alpha+\theta<2$.

For $\theta=1$, our set-up coincides with the Greenwood model (Ref. $^{[3]}$, p. 71) of contagious disease: The connection is made by identifying an available component with a healthy individual and an unavailable component with an infected individual.

Our assumptions allow the theory of Markov chains to be brought to bear. We take the number of components available to be the state of our chain. The transition probability $p_{i, j}$ of moving from state $j$ to state $i$ in one unit of time is readily computed. Such a transition results when $l$ of the $j$ available components remain in service and $i-l$ of the $n-j$ unavailable components are returned to service. Thus,

$$
p_{i, j}=\sum_{l=0}^{i}\left(\begin{array}{l}
j \\
l
\end{array}\right)\left(\begin{array}{c}
n-j \\
i-l
\end{array}\right) \alpha^{l} \underline{\alpha}^{j-l} \theta^{n+l-i-j} \underline{\theta}^{i-l} .
$$

Among many results aimed at measuring the reliability of an $n$-component system, we present but three (under our assumptions, of course):

R1 The expected number of components available at time $t$ is $\frac{n\left(\underline{\theta}+\underline{\alpha} q^{t}\right)}{1-q}$ where, for convenience, $q=\alpha+\theta-1$.

R2 The expected time it takes for the system to crash (that is, all components are out of service) is $\left(\frac{1-q}{\underline{\alpha}}\right)^{n}+\sum_{k=1}^{n}\left(\begin{array}{l}n \\ k\end{array}\right)\left((\underline{\theta} / \underline{\alpha})^{k}-\right.$ $\left.(-1)^{k}\right) \frac{q^{k}}{1-q^{k}}$.

R3 As time $t \rightarrow \infty$, the expected fraction of time for which $i$ of the $n$ components are available approaches $\left(\begin{array}{l}n \\ i\end{array}\right) \frac{\underline{\alpha}^{n-i} \underline{\theta}^{i}}{(1-q)^{n}}$.

We begin with a brief discussion of a relevant class of matrices.

\section{VANDERMONDE MATRICES}

For convenience, we set

$$
V_{i, j}\left(\begin{array}{l}
a, b \\
c, d
\end{array}\right)=\sum_{l=0}^{i}\left(\begin{array}{l}
j \\
l
\end{array}\right)\left(\begin{array}{c}
n-j \\
i-l
\end{array}\right) a^{n+l-i-j} b^{j-l} c^{i-l} d^{l}
$$

We then define the $n$th Vandermonde matrix with parameters $a, b, c$, and $d$ to be the array

$$
\left[\begin{array}{ll}
a & b \\
c & d
\end{array}\right]_{n}=\left(V_{i, j}\left(\begin{array}{l}
a, b \\
c, d
\end{array}\right)\right)_{0 \leq i, j \leq n}
$$


For $n=3$,

$$
\left[\begin{array}{ll}
a & b \\
c & d
\end{array}\right]_{3}=\left(\begin{array}{cccc}
a^{3} & a^{2} b & a b^{2} & b^{3} \\
3 a^{2} c & 2 a b c+a^{2} d & b^{2} c+2 a b d & 3 b^{2} d \\
3 a c^{2} & b c^{2}+2 a c d & 2 b c d+a d^{2} & 3 b d^{2} \\
c^{3} & c^{2} d & c d^{2} & d^{3}
\end{array}\right)
$$

In Ref. ${ }^{[5]}$, we proved the following remarkable fact.

Theorem 2.1. If $a, b, \ldots, h$ are elements of a field, then

$$
\left[\begin{array}{ll}
a & b \\
c & d
\end{array}\right]_{n}\left[\begin{array}{ll}
e & f \\
g & h
\end{array}\right]_{n}=\left[\left(\begin{array}{ll}
a & b \\
c & d
\end{array}\right)\left(\begin{array}{ll}
e & f \\
g & h
\end{array}\right)\right]_{n} .
$$

In other words, the product of two Vandermonde matrices is Vandermonde. Moreover, the matrix of parameters for the product miraculously coincides with the product of the underlying two-by-two matrices of parameters!

Theorem 2.1 allows us to multiply, invert, and diagonalize Vandermonde matrices at will. For instance, if $a d-b c \neq 0$, then Theorem 2.1 implies that

$$
\left[\begin{array}{ll}
a & b \\
c & d
\end{array}\right]_{n}^{-1}=\left[\begin{array}{cc}
\frac{d}{a d-b c} & \frac{-b}{a d-b c} \\
\frac{-c}{a d-b c} & \frac{a}{a d-b c}
\end{array}\right]_{n}=\frac{1}{(a d-b c)^{n}}\left[\begin{array}{cc}
d & -b \\
-c & a
\end{array}\right]_{n} .
$$

The relevance of Vandermonde matrices for our present intentions should be apparent: In view of (1) and (2), $p_{i, j}=V_{i, j}\left(\begin{array}{l}\theta, \underline{\alpha}, \underline{\alpha}) \\ \theta\end{array}\right)$ So our transition matrix

$$
P=\left(p_{i, j}\right)_{0 \leq i, j \leq n}=\left[\begin{array}{cc}
\theta & \underline{\alpha} \\
\underline{\theta} & \alpha
\end{array}\right]_{n}
$$

is Vandermonde.

\section{MULTI-STEP TRANSITION PROBABILITIES AND R1}

Determination of the probability $p_{i, j}^{(t)}$ of moving from state $j$ to state $i$ in $t$ units of time is key in deducing the results $\mathbf{R 1}, \mathbf{R 2}$, and $\mathbf{R 3}$. To this end, we diagonalize our transition matrix $P$ in (4) (which, by Theorem 2.1, is no more difficult than diagonalizing a $2 \times 2$ matrix). Let

$$
Q=\left[\begin{array}{cc}
\underline{\alpha} & -1 \\
\underline{\theta} & 1
\end{array}\right]_{n} \text { and } D=\left[\begin{array}{ll}
1 & 0 \\
0 & q
\end{array}\right]_{n}
$$


Then, Theorem 2.1 and (3) together imply that

$$
Q^{-1} P Q=D
$$

Corollary 3.1. If $0<\alpha+\theta<2$, then the probability of moving from state $j$ to state $i$ in $t$ units of time is given by

$$
p_{i, j}^{(t)}=\frac{1}{(1-q)^{n}} \sum_{l=0}^{j}\left(\begin{array}{l}
j \\
l
\end{array}\right)\left(\begin{array}{c}
n-j \\
i-l
\end{array}\right) a^{n+l-i-j} b^{j-l} c^{i-l} d^{l}
$$

where

$$
a=\underline{\alpha}+\underline{\theta} q^{t}, \quad b=\underline{\alpha}\left(1-q^{t}\right), \quad c=\underline{\theta}\left(1-q^{t}\right), \quad \text { and } \quad d=\underline{\theta}+\underline{\alpha} q^{t} .
$$

Moreover, if the chain begins in state $j$, then the state probability generating function at time $t$ is

$$
\sum_{i=0}^{n} p_{i, j}^{(t)} z^{i}=\frac{(a+c z)^{n-j}(b+d z)^{j}}{(1-q)^{n}}
$$

Proof. As the multi-step transition probability $p_{i, j}^{(t)}$ is the $i j$ th entry in $P^{t}$, we apply (5) and Theorem 2.1 to obtain

$$
P^{t}=Q D^{t} Q^{-1}=\frac{1}{(1-q)^{n}}\left[\begin{array}{ll}
a & b \\
c & d
\end{array}\right]_{n}
$$

where $a, b, c$, and $d$ are as in (7). Hence, (6) now follows from (2).

For (8), observe that (6) implies that

$$
\sum_{i=0}^{n} p_{i, j}^{(t)} z^{i}=\frac{1}{(1-q)^{n}} \sum_{l=0}^{j}\left(\begin{array}{l}
j \\
l
\end{array}\right) b^{j-l}(d z)^{l} \sum_{i=l}^{n-j+l}\left(\begin{array}{c}
n-j \\
i-l
\end{array}\right) a^{(n-j)-(i-l)}(c z)^{i-l} .
$$

Two applications of the binomial theorem then gives (8).

Derivation of $\mathbf{R} \mathbf{1}$ is now a routine matter from (8): Taking $j=n$, differentiating with respect to $z$, and then setting $z=1$ does the job.

\section{A WAITING TIME DISTRIBUTION AND R2}

To verify R2, we first consider how long it takes for a Markov chain to travel from one state to another. Let $f_{i, j}^{(t)}$ denote the probability that the chain visits state $i$ for the first time at time $t$ given that the process begins in state $j$. For $i=j, f_{i, i}^{(t)}$ is the probability of returning to state $i$ for the first 
time after $t$ steps. The fundamental relationship between the waiting time probabilities $f_{i, j}^{(t)}$ and the multi-step probabilities $p_{i, j}^{(t)}$ is as follows.

Theorem 4.1 (First Entrance Theorem). If we let

$$
M_{i, j}(z)=(1-z) \sum_{t \geq 1} p_{i, j}^{(t)} z^{t} \quad \text { and } \quad F_{i, j}(z)=\sum_{t \geq 1} f_{i, j}^{(t)} z^{t}
$$

then

$$
F_{i, j}(z)=\frac{M_{i, j}(z)}{1-z+M_{i, i}(z)}
$$

for $|z|<1$. Moreover, if

$$
p_{i}^{(\infty)}=\lim _{t \rightarrow \infty} p_{i, j}^{(t)} \text { exists, is independent of } j \text {, and is positive, }
$$

then $F_{i, j}$ is a probability generating function, that is, $F_{i, j}$ is left-continuous at $z=1$ with $F_{i, j}(1)=1$.

Proof. As in Ref. ${ }^{[3]}$, p. 89,

$$
f_{i, j}^{(t)}=p_{i, j}^{(t)}-\sum_{k=1}^{t-1} f_{i, j}^{(k)} p_{i, i}^{(t-k)}
$$

for all $t \geq 1$. Multiplying (11) by $z^{t}$ and summing over $t \geq 1$ gives a formula equivalent to $(9)$ :

$$
F_{i, j}(z)=\sum_{t \geq 1} p_{i, j}^{(t)} z^{t}-F_{i, j}(z) \sum_{t \geq 1} p_{i, i}^{(t)} z^{t}
$$

Next, suppose that (10) holds. For $|z|<1$, observe that

$$
M_{i, j}(z)=\sum_{t \geq 1}\left(p_{i, j}^{(t)}-p_{i, j}^{(t-1)}\right) z^{t}
$$

where $p_{i, j}^{(0)}=0$. As the series

$$
\sum_{t \geq 1}\left(p_{i, j}^{(t)}-p_{i, j}^{(t-1)}\right)
$$

telescopes to $p_{i}^{(\infty)}$, it follows from Abel's Theorem that $M_{i, j}(z)$ is leftcontinuous at $z=1$ with $M_{i, j}(1)=p_{i}^{(\infty)}$. Thus, $\lim _{z \rightarrow 1^{-}} F_{i, j}(z)=1$.

We now take aim at our second result R2. First, note that it is correct when $\alpha=1$ : In this case, the expected crash time in infinite. So consider 
the case $\alpha<1$. As we are also assuming that $0<\alpha+\theta<2$, it follows that $|q|<1$. Corollary 3.1 then guarantees that (10) holds for $i=0$. Thus, $F_{0, n}(z)$ is a probability generating function. Also by Corollary 3.1,

$$
p_{0, n}^{(t)}=\left(\frac{\underline{\alpha}\left(1-q^{t}\right)}{1-q}\right)^{n} \quad \text { and } \quad p_{0,0}^{(t)}=\left(\frac{\underline{\alpha}+\underline{\theta} q^{t}}{1-q}\right)^{n} .
$$

Note that $M_{0, n}(1)=\underline{\alpha}^{n} /(1-q)^{n}=M_{0,0}(1)$. With the aid of (12), (13), the extended binomial theorem, and a little finagling, we obtain

$$
\begin{aligned}
M_{0, n}^{\prime}(1) & =\underline{\alpha}^{n}+\left(\frac{\underline{\alpha}}{1-q}\right)^{n} \sum_{t \geq 2} t\left(\sum_{k=1}^{n}(-1)^{k}\left(\begin{array}{l}
n \\
k
\end{array}\right)\left(q^{k}-1\right) q^{k(t-1)}\right) \\
& =\left(\frac{\underline{\alpha}}{1-q}\right)^{n}\left(1+\sum_{k=1}^{n}(-1)^{k-1}\left(\begin{array}{l}
n \\
k
\end{array}\right) \frac{q^{k}}{1-q^{k}}\right) .
\end{aligned}
$$

Similarly,

$$
M_{0,0}^{\prime}(1)=\left(\frac{\underline{\alpha}}{1-q}\right)^{n}\left(1-\sum_{k=1}^{n}\left(\begin{array}{l}
n \\
k
\end{array}\right)(\underline{\theta} / \underline{\alpha})^{k} \frac{q^{k}}{1-q^{k}}\right) .
$$

Finally, applying logarithmic differentiation to (9) yields R2:

$$
\begin{aligned}
F_{0, n}^{\prime}(1) & =\frac{M_{0, n}^{\prime}(1)}{M_{0, n}(1)}-\frac{M_{0,0}^{\prime}(1)-1}{M_{0,0}(1)} \\
& =\left(\frac{1-q}{\underline{\alpha}}\right)^{n}+\sum_{k=1}^{n}\left(\begin{array}{l}
n \\
k
\end{array}\right)\left((-1)^{k-1}+(\underline{\theta} / \underline{\alpha})^{k}\right) \frac{q^{k}}{1-q^{k}} .
\end{aligned}
$$

In the context of $n$-player Russian roulette, the case $\theta=1$ of $\mathbf{R 2}$ is discussed in Rawlings ${ }^{[4]}$. Bartholdi ${ }^{[1]}$ considered a variation of $\mathbf{R 2}$ in which $n$ lamps are turned on and off according to a set of deterministic rules.

\section{THE EXPECTED NUMBER OF VISITS AND R3}

To get at our final result R3, we consider how often a given state is visited on a fixed time interval. This issue is resolved by Theorem 5.1 (a proof of which may be found in Ref. ${ }^{[2]}$, p. 105).

Theorem 5.1. If a Markov chain begins in state $j$, then the expected number of times state $i$ is visited on the interval $[1, \tau]$ is $\sum_{t=1}^{\tau} p_{i, j}^{(t)}$. 
For R3, we again assume $0<\alpha+\theta<2$ and that the chain begins in state $n$. If $\theta=1$ (so $\alpha<1$ ), then $\mathbf{R 3}$ is easily seen to be correct: It's 1 if $i=0$ and 0 if $i>1$. So assume that $\theta<1$. Then, by Corollary 3.1,

$$
p_{i, n}^{(t)}=\left(\begin{array}{c}
n \\
i
\end{array}\right) \frac{\underline{\alpha}^{n-i} \underline{\theta}^{i}}{(1-q)^{n}}\left(1-q^{t}\right)^{n-i}\left(1+\frac{\underline{\alpha} q^{t}}{\underline{\theta}}\right)^{i} .
$$

So, relative to the time interval $[1, \tau]$, Theorem 5.1 implies that the expected fraction of the time for which our system has $i$ components available is

$$
\frac{1}{\tau} \sum_{t=1}^{\tau}\left(\begin{array}{c}
n \\
i
\end{array}\right) \frac{\underline{\alpha}^{n-i} \underline{\theta}^{i}}{(1-q)^{n}}\left(1-q^{t}\right)^{n-i}\left(1+\frac{\underline{\alpha} q^{t}}{\underline{\theta}}\right)^{i} .
$$

As $\lim _{\tau \rightarrow \infty} \frac{1}{\tau} \sum_{t=1}^{\tau} \prod_{k=1}^{n}\left(1+a_{k} q^{t}\right)=1$ when $a_{1}, a_{2}, \ldots, a_{n}$ are real and $|q|<1$, letting $\tau \rightarrow \infty$ in (14) gives R3.

As an exercise, Viswanadham and Narahari ${ }^{[6]}$ (p. 206) pose the problem of determining the asymptotic expected fraction of time for which at least $m$ of the $n$ components are available when $n=3$ and when unavailable components are returned to service according to a certain deterministic rule. Under our assumptions, $\mathbf{R 3}$ gives the solution to their problem for any non-negative integer $n$ as $\sum_{i=m}^{n}\left(\begin{array}{l}n \\ i\end{array}\right) \underline{\alpha}^{n-i} \underline{\theta}^{i} /(1-q)^{n}$.

\section{REFERENCES}

1. Bartholdi, L. Lamps, factorizations, and finite fields. Amer. Math. Monthly 1999, 107, 429-436.

2. Bhat, U.N. Elements of Applied Stochastic Processes; 2nd ed.; John Wiley: New York, 1984.

3. Iosifescu, M. Finite Markov Processes and Their Applications; Wiley: New York, 1980.

4. Rawlings, D. Sequential searches: Proofreading, Russian roulette, and the incomplete $q$-Eulerian polynomials revisited. Amer. Math. Monthly 2001, 108, 713-720.

5. Rawlings, D.; Sze, L. On the metamorphosis of Vandermonde's identity. Math. Mag. 2005, June, 232-238.

6. Viswanadham, N.; Narahari, Y. Performance Modeling of Automated Manufacturing Systems; New Jersey: Prentice Hall, 1992. 